\title{
Artritis como debut de enfermedad inflamatoria intestinal en pediatría
}

\author{
Arthritis as clinical presentation of inflammatory bowel disease in children
}

\author{
Dra. Inés Félez Molinera, Dra. Ruth García Romero ${ }^{a}$, Dr. Ignacio Ros Arnal ${ }^{a}$, Dr. Marcos Clavero Adella \\ Dr. Eduardo Ubalde Sainz y Dra. Marta Medrano San Ildelfonso ${ }^{a}$
}

\begin{abstract}
RESUMEN
La incidencia de enfermedad inflamatoria intestinal en la edad pediátrica se ha incrementado mundialmente en las últimas décadas. La forma de presentación puede ser diversa y, hasta en un 6-35\%, las manifestaciones extraintestinales pueden ser el debut; la artritis periférica es la más frecuente de estas. Una presentación atípica implica un retraso diagnóstico y, asociado a que el fenotipo de enfermedad inflamatoriaintestinal es más grave en los niños, conlleva un incremento de las complicaciones intestinales y secuelas asociadas. Se presentan dos casos clínicos de enfermedad de Crohn cuya clínica inicial fue la claudicación de la marcha por una artritis periférica y una entesitis, respectivamente.

Palabras clave: enfermedades inflamatorias intestinales, artritis periférica, entesitis, enfermedad de Crohn.
\end{abstract}

\begin{abstract}
Inflammatory bowel disease in children has increased worldwide during the last decades. Clinical presentations are diverse and extraintestinal manifestations are the presenting sign in 6-35\% of patients, the most common of them being peripheral arthritis. An atypical clinical presentation results in diagnosis delay and, added to the greater seriousness of inflammatory bowel disease phenotypes in children, it entails more intestinal complications and sequelae. We describe two cases of inflammatory bowel disease with an initial symptom of lameness due to peripheral arthritis and enthesitis, respectively. Key words: inflammatory bowel disease, peripheral arthritis, enthesitis, Crohn's disease.
\end{abstract}

http:/ / dx.doi.org/10.5546/ aap.2019.e270

Cómo citar: Félez Moliner I, García Romero R, Ros Arnal I, Clavero Adell M, et al. Artritis como debut de enfermedad inflamatoria intestinal en pediatría. Arch Argent Pediatr 2019;117(3):e270-e273.

a. Hospital Universitario Miguel Servet. Zaragoza, España.

Correspondencia:

Dra. Inés Félez Moliner: inesfelez@gmail.com

Financiamiento: Ninguno.

Conflicto de intereses: Ninguno que declarar.

Recibido: 8-7-2018

Aceptado: 20-12-2018

\section{INTRODUCCIÓN}

La incidencia de la enfermedad inflamatoria intestinal (EII) en la edad pediátrica se ha incrementado mundialmente en las últimas décadas. ${ }^{1}$ A pesar de que no hay estimaciones exactas, el registro español SPIRIT (siglas de Spanish Pediatric IBD Restrospective study of Incidence Trends) observó que la incidencia de la EII se había incrementado del 0,97 al 2,8/100000 en el período de 1996-2009, y era mayor el aumento de incidencia en la enfermedad de Crohn (EC). ${ }^{2}$

La forma de presentación es variada y puede iniciarse con clínica digestiva, síntomas inespecíficos (como pérdida de peso, retraso del crecimiento y anemia) o con manifestaciones extraintestinales, sobre todo en la EC. ${ }^{1,3}$

Las manifestaciones extraintestinales son diversas: afectación músculo-esquelética (artritis, espondilitis, sacroileítis, entesitis, dactilitis, osteopenia, osteoporosis), ocular (uveítis y epiescleritis), cutánea (eritema nodoso, pioderma gangrenoso), hepatobiliar (colangitis esclerosante primaria, hepatitis autoinmune) o hematológica (anemia, enfermedad tromboembólica). ${ }^{3}$

Dentro de estas, la más frecuente es la artritis periférica y se caracteriza por su escasa correlación con el haplotipo HLA-B27 y por presentar factor reumatoide negativo. En la afectación axial, sin embargo, el HLA-B27 es positivo en el $60 \%$ de los casos. ${ }^{4,5}$

Se exponen dos casos de EII cuya forma de presentación y razón de ingreso fue la claudicación de la marcha, causada, en un caso, por entesitis $y$, en otro, por artritis periférica.

\section{CASOS CLÍNICOS \\ Caso clínico 1}

Se trata de un paciente varón de 9 años que ingresó por presentar cojera de 3 semanas de evolución, con dolor en los muslos, en los gemelos de ambas extremidades y en el pie derecho, asociado a diarrea y vómitos desde el inicio. Había estado ingresado en otro centro hospitalario hacía cuatro años por un cuadro similar sin llegar 
a un diagnóstico claro. Desde entonces, había presentado tres episodios similares (artralgias y diarrea) tratados con pauta corta de corticoides orales por su pediatra. En la exploración, se destacó fenotipo delgado (-1,5 desvíos estándar DE-), aftas orales y dificultad en la deambulación con signos inflamatorios del tendón de Aquiles derecho. Dada la clínica articular, se realizó una gammagrafía ósea, en la que se visualizó un aumento del pool vascular en la región supramaleolar del pie derecho, y una ecografía articular, en la que se observó un engrosamiento e hiperecogenicidad de la región preaquílea derecha. El estudio de autoinmunidad resultó negativo. Ante la clínica digestiva asociada, se realizó una analítica y se observó anemia (hemoglobina: 9,5 g/dl) y elevación de reactantes de fase aguda (proteína $C$ reactiva -PCR-: 14,40 mg/dl; velocidad de sedimentación glomerular -VSG-: $77 \mathrm{~mm} / \mathrm{h}$ ); una ecografía abdominal, en la que se observó el engrosamiento parietal de todo el colon, y una endoscopia digestiva (Figura 1), en la que se visualizaron úlceras en el esófago y úlceras profundas parcheadas con pseudopólipos en el colon, hallazgos compatibles con EC de larga evolución.

\section{Caso clínico 2}

Se trata de una paciente mujer de 6 años que ingresó por la claudicación de la marcha de dos semanas de evolución. Asociaba pérdida de peso desde hacía dos meses sin clínica digestiva. En la exploración física, se destacó dolor ante la movilización y la palpación del tobillo y del tarso del pie derecho con signos inflamatorios. Se realizó una gammagrafía ósea, en la que se visualizó una lesión vascularizada en el tarso derecho, y un estudio de autoinmunidad, que fue negativo. Dos semanas después, ingresó por presentar un empeoramiento de la clínica articular y diarrea sanguinolenta. En la analítica, se destacó anemia (hemoglobina: $9 \mathrm{~g} / \mathrm{dl}$ ) y elevación de reactantes de fase aguda (PCR: $16,42 \mathrm{mg} / \mathrm{dl}$; VSG: $68 \mathrm{~mm}$ /hora). Se realizó una ecografía abdominal, en la que se identificó un engrosamiento mural del colon ascendente y transverso, y una endoscopía digestiva (Figura 2), en la que se observaron lesiones aftosas en el duodeno, el íleon distal y el colon compatibles con EC.

Ambos casos presentaron clínica músculoesquelética (entesitis y artritis periférica), asociada a EC de difícil control, que precisó el tratamiento con medicación anti factor de necrosis tumoral

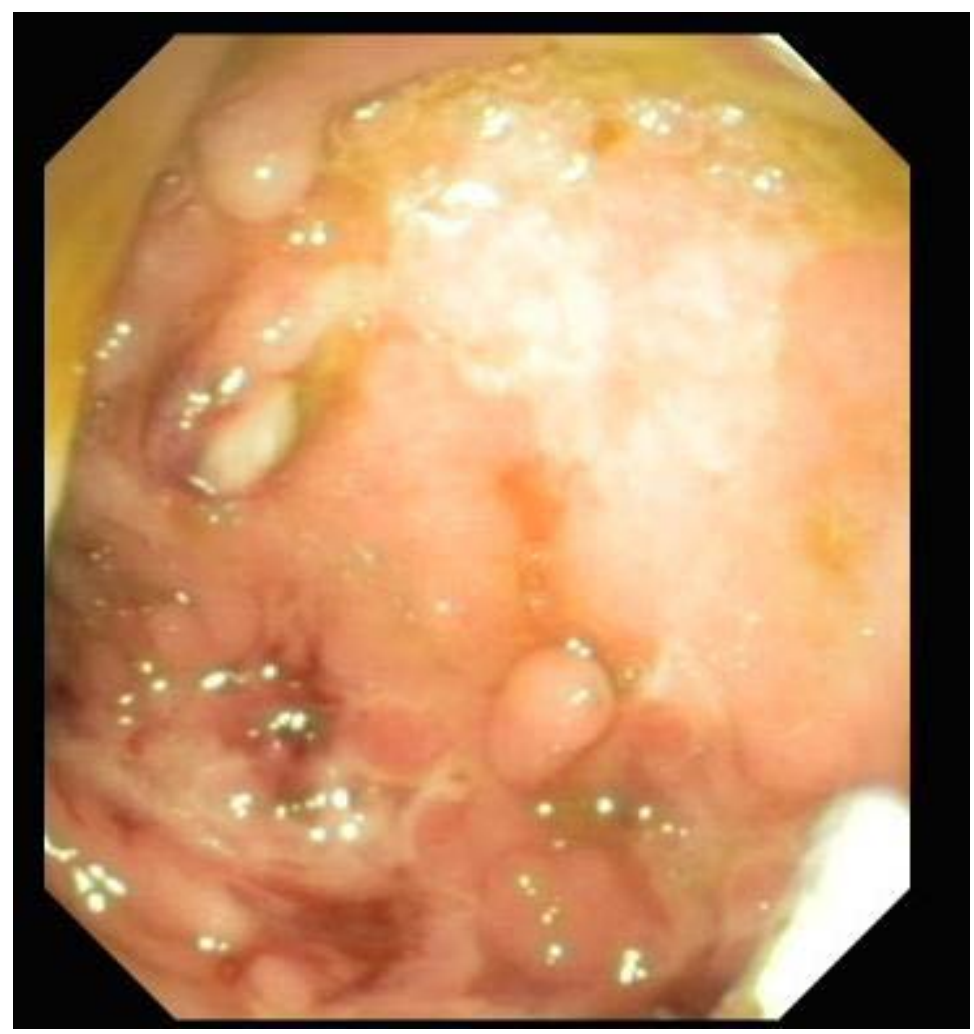


(anti-tumor necrosis factor; anti-TNF, por sus siglas en inglés) (adalimumab), que consiguió una mejoría progresiva de la clínica, tanto digestiva como articular.

\section{DISCUSIÓN}

El retraso diagnóstico de la EII es frecuente en los niños, debido, principalmente, a una presentación atípica en el $21 \%$ de los casos. Las manifestaciones extraintestinales como clínica inicial se dan en el 6-35\% de los pacientes. ${ }^{6}$ El estudio de Kwon et al. observó un retraso diagnóstico de 3,4 meses en los niños con EC. ${ }^{7}$ En el primero de nuestros casos, el retraso diagnóstico fue de cuatro años.

Dentro de las manifestaciones extraintestinales, la afectación articular es la más frecuente, tanto en los niños como en los adultos, con una incidencia del 16-33\%., Esta puede presentarse con afectación periférica en forma de artritis (el 7-16\%), entesitis (el 5-10\%), dactilitis (el 2-4 \%) o artralgias (el 8-30\%), o con afectación axial como espondilitis (el 1-10\%), sacroileítis (el 2-32\%) o dolor inflamatorio (el 5-30\%)., ${ }^{4,5}$

El momento de presentación de la artritis periférica es variable, aunque suele ocurrir tras el diagnóstico de la EII. Según los resultados de distintos estudios, solo el 15-18\% de estas se presentan antes del diagnóstico. En cambio, el inicio de la afectación axial suele ser independiente y, con frecuencia, precede la EII. ${ }^{9}$

La artritis periférica es la artropatía más frecuente y se puede diferenciar en dos tipos según los criterios definidos por Orchard et al. ${ }^{10}$ El tipo $1 \mathrm{u}$ oligoarticular asimétrico suele tratarse de episodios que afectan a menos de cinco articulaciones y coincide con exacerbaciones de la EII. Además, asocia frecuentemente otras manifestaciones extraintestinales. El tipo 2 o poliarticular suele ocasionar episodios de meses o años de duración que involucran cinco o más articulaciones y tienen una evolución independiente de la EII. Este segundo tipo no se asocia con manifestaciones extraintestinales, salvo la uveítis. Ambos tipos de artritis periférica se caracterizan por ser no erosivos ni deformantes, aunque un $10 \%$ de los pacientes desarrollan erosiones.

El paciente del primer caso presentó una entesitis del tendón de Aquiles. Esta y la fascitis plantar son las dos presentaciones clínicas más frecuentes de la entesitis. ${ }^{5}$ La paciente del

FIGURA 2. Lesiones aftosas e hiperémicas dispersas, que alternan con zonas de mucosa normal en el colon

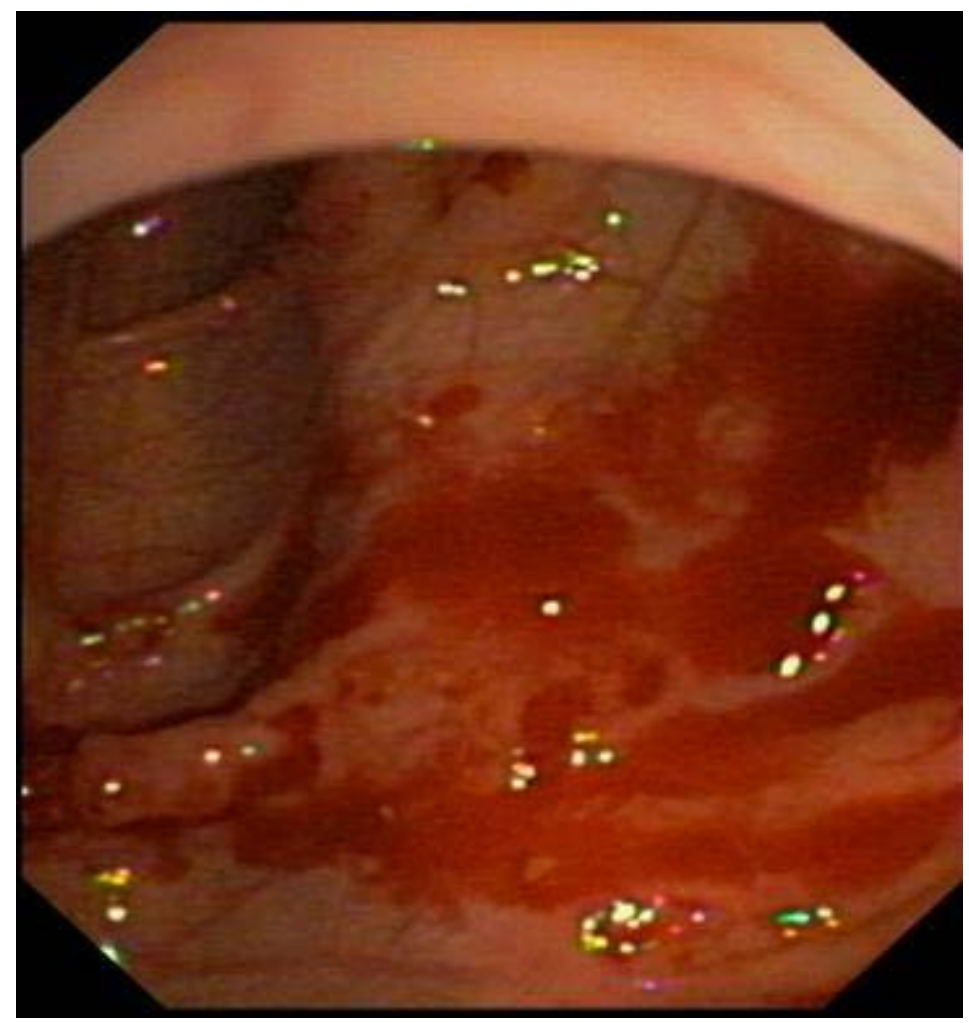


segundo caso debutó con oligoartritis asimétrica de los miembros inferiores, con afectación del tobillo y del tarso izquierdos, con respuesta incompleta al adalimumab. Precisó infiltración local y la intensificación del tratamiento, y se añadió metotrexato al adalimumab.

El curso de la EC con inicio en la infancia es más agresivo que el fenotipo de la edad adulta y presenta, con mayor frecuencia, complicaciones y secuelas a largo plazo. En más de un tercio de los niños con EII, se producen complicaciones intestinales en un período menor de diez años desde el diagnóstico. ${ }^{11}$ Una de las principales secuelas es el retraso del crecimiento, que ocurre en el $40 \%$ de los pacientes pediátricos con EC, así como deficiencias nutricionales. Además, los niños afectos presentan peor calidad de vida y mayor prevalencia de ansiedad o depresión en comparación con los niños afectos de otras enfermedades crónicas. ${ }^{3}$ En cuanto a la mortalidad y a la incidencia de cáncer, no hay resultados claros, pero parece que el riesgo relativo de ambos se encuentra incrementado en estos pacientes. ${ }^{11}$ Por estas razones, una alta sospecha clínica es esencial para un diagnóstico y tratamiento precoces, dado que puede mejorar la evolución y el pronóstico de esta enfermedad.

\section{REFERENCIAS}

1. Oliveira SB, Monteiro IM. Diagnosis and management of inflammatory bowel disease in children. BMJ. 2017; 357:j2083.

2. Martín-de-Carpi J, Rodríguez A, Ramos E, Jiménez S, et al. The complete picture of changing pediatric inflammatory bowel disease incidence in Spain in 25 years (1985-2009): the EXPERIENCE registry. J Crohns Colitis. 2014; 8(8):763-9.

3. Rosen MJ, Dhawan A, Saeed SA. Inflammatory Bowel Disease in Children and Adolescents. JAMA Pediatr. 2015; 169(11):1053-60.

4. BrakenhoffLK, van der HeijdeDM,Hommes DW, Huizinga TWJ, Fidder HH. The joint-gut axis in inflammatory bowel diseases. J Crohns Colitis. 2010; 4(3):257-68.

5. Salvarani C, Fries W. Clinical features and epidemiology of spondyloarthritides associated with inflammatory bowel disease. World J Gastroenterol. 2009; 15(20):2449-55.

6. Yu YR, Rodriguez JR. Clinical presentation of Crohn's, ulcerative colitis, and indeterminate colitis: Symptoms, extraintestinal manifestations, and disease phenotypes. Semin Pediatr Surg. 2017; 26(6):349-55.

7. Kwon YH, Kim YJ. Pre-diagnostic Clinical Presentations and Medical History Prior to the Diagnosis of Inflammatory Bowel Disease in Children. Pediatr Gastroenterol Hepatol Nutr. 2013; 16(3):178-84.

8. Cardile S, Romano C. Current issues in pediatric inflammatory bowel disease-associated arthropathies. World J Gastroenterol. 2014; 20(1):45-52.

9. Paredes JM, Barrachina MM, Román J, Moreno-Osset E. Patología articular en la enfermedad inflamatoria intestinal. Gastroenterol Hepatol. 2005; 28(4):240-9.

10. Orchard TR, Wordsworth BP, Jewell DP. Peripheral arthropathies in inflammatory bowel disease: their articular distribution and natural history. Gut. 1998; 42(3):387-91.

11. Duricova D, Fumery M, Annese V, Lakatos PL, et al. The natural history of Crohn's disease in children: a review of population-based studies. Eur J Gastroenterol Hepatol. 2017; 29(2):125-34 